\title{
Amount of training effects in representation- mediated food aversion learning: No evidence of a role for associability changes
}

\author{
PETER C. HOLLAND \\ Johns Hopkins University, Baltimore, Maryland
}

\begin{abstract}
Rats acquired aversions to food pellets when a previously trained signal for that food was paired with a toxin, but only after minimal signal-food training. After extensive signal-food training, signal-toxin pairings had no effect on food consumption even after manipulations that enhanced the associability of the signal. By contrast, conditioned responding to the signal retained its sensitivity to devaluation of the food reinforcer by food-toxin pairings after extensive training. These results suggest that the nature of associatively activated event representations changes over the course of training.
\end{abstract}

According to most modern descriptions of Pavlovian conditioning, pairings of a conditioned stimulus (CS) and an unconditioned stimulus (US) endow the CS with the ability to activate a memorial representation of the US. One consequence of this activation is that the CS often comes to elicit a conditioned response (CR) that shares features with the unconditioned response. Considerable evidence indicates that CS activation of US representations may serve a number of functions beyond determination of $\mathrm{CR}$ form, influencing performance in a variety of learning tasks, including reinforcer revaluation (see, e.g., Balleine \& Dickinson, 1998; Holland \& Rescorla, 1975), mediated learning (Dwyer, 2003; Hall, 1996; Holland, 1981, 1990), differential outcome expectancy (see, e.g., Blundell, Hall, \& Killcross, 2001; Rescorla, 1992; Trapold \& Overmier, 1972), and acquired equivalence (Hall, 1996).

Hall (1996) and Holland (1990) described a number of contexts in which associatively activated event representations appear to substitute for their referents in the control of learning and action. Perhaps the most commonly investigated instance is that of reinforcer revaluation, in which the reinforcement value of the US is changed after the completion of CS-US training. For example, Holland and Rescorla (1975) and Holland and Straub (1979) first presented rats with pairings of a tone $\mathrm{CS}$ with a food pellet US and then paired access to the food pellets in the home cage with the induction of illness. Subsequent tests with the tone alone showed significant reductions in CRs relative to the performance of rats that received food and illness unpaired. In experiments such as these, performance

This research was supported by Grant MH65879 from the National Institutes of Health. I thank Erin Kerfoot and Vanessa McKenna for their technical assistance. Correspondence concerning this article may be addressed to P. C. Holland, Johns Hopkins University, 204 Ames Hall, 3400 North Charles Street, Baltimore, MD 22128 (e-mail: pch@jhu.edu). of CRs is thought to be mediated by a CS-activated representation of the US, which substitutes for a representation activated by the US itself in the control of behavior. Posttraining changes in the value of the US representation then could result in spontaneous changes in CRs elicited by the CS.

Hall (1996) and Holland (1981, 1990) showed that associatively activated US representations may also substitute for their referents in the acquisition of new learning. For example, in several experiments (Holland, 1981, 1990) in which a variety of auditory or visual CSs and solid or liquid food USs were used, rats first received CS-food pairings. Then, in the absence of the food US, the CS was either paired with toxin-induced illness or presented explicitly unpaired with that illness. Finally, consumption of the food in the absence of the CS was assessed. Rats that received CS-illness pairings consumed less food than rats that received CS and illness unpaired. Holland (1981) claimed that the learned food aversion was mediated by a CS-activated representation of the food: Pairing of that representation of the food with illness was sufficient to establish an aversion to the food itself.

Later experiments (Holland, 1990, Experiment 10; 1998, Experiments 1 and 2) showed a limitation in the conditions under which this mediated food aversion learning occurred-specifically, in the number of CS-food pairings in the initial training phase. In those experiments, mediated food aversion learning was observed only after relatively small numbers (16-20) of CS-food pairings; after as few as 40 pairings, there was no evidence of representation-mediated food aversion learning. A simple account for this outcome is that CSs lose the ability to activate a US representation as conditioning proceeds, and CRs are instead increasingly elicited by stimulus-response habits (see, e.g., Adams \& Dickinson, 1981; Bussey, Muir, Everitt, \& Robbins, 1996; Kimble \& Perlmuter, 1970; Poldrack \& Packard, 2003). However, comparable studies of the effects of amount of training on sensitivity of Pavlov- 
ian CRs to reinforcer revaluation procedures (Holland, 1998, Experiment 3) showed that representation-mediated performance of CRs was unaffected by the amount of initial CS-food training in this conditioning procedure: Responding to the CS was equally reduced by posttraining food-toxin pairings after 16 and after $160 \mathrm{CS}$-food pairings. Thus, although extensive CS-food training reduced rats' use of an associatively activated representation of food to establish new learning about that food, it had no effect on the mediation of CR performance by such a representation.

To accommodate this dissociation between the effects of amount of training on mediated learning and reinforcer devaluation, Holland (1998) proposed that the nature of associatively activated event representations changes over the course of learning. Although the notion of an associatively activated US representation is frequently invoked in theoretical descriptions of conditioning, the nature of such representations is seldom specified. Holland (1990) suggested that an associatively activated event representation refers simply to a set of nervous system processes normally evoked by the US, which come under the control of a CS through learning. Early in training, CSs may access a different subset of these processes than they do later in training. For example, Holland (1998) suggested that early in training CSs may activate perceptual processing normally activated only by the US, but are unable to do so later in training. Casually speaking, early in training rats may functionally taste the food in the presence of the CS prior to food delivery itself. Thus, when a minimally trained CS for food is paired with toxin, perceptual processing of the food flavor, like that produced by presentation of the food itself, is paired with illness, establishing a flavor-illness association. According to this view, the CS alone loses its ability to activate perceptual processing of the upcoming food as conditioning proceeds, and so CS-illness pairings would not provide the taste-illness pairings required for taste aversion learning. By contrast, the sensitivity of previously established CRs to reinforcer revaluation may be mediated by less transient aspects of an associatively activated US representation, which incorporate the US's incentive value but do not provoke perceptual processing of that event in its absence.

However, a much simpler account for both the loss of mediated aversion learning and the maintenance of reinforcer devaluation effects with extensive training in Holland's (1998) experiments may be provided by extension of a model of conditioning proposed by Pearce and Hall (1980). In that model, the learning rate parameter $(\alpha$, termed associability) of a CS declines as its consequences become more reliably predicted. With simple pairings of a single CS with a US, as that CS becomes a better predictor of the US, its associability declines. Thus, the more CS-US pairings, the less the CS will be able to acquire new learning. Indeed, in this model a major determinant of negatively accelerated learning curves is the gradual loss of a CS's associability as it becomes a more reliable predictor of the US. Notably, in this model CS associabil- ity is assumed to influence only learning and not performance; the ability of a CS to elicit CRs, once they are established, is not influenced by its associability. Thus, CSs maintain their ability to elicit CRs even as they lose the ability to acquire new learning.

When this model is applied to Holland's (1998) experiments, it could be argued that extensive training of a CS reduces not only the ability of the same CS to enter into new associations, but also the ability of any representation that the CS activates. Thus, with extensive training of a CS, a CS-activated representation of food would lose its ability to enter into new associations with illness. At the same time, because the associability of a CS does not affect its previously established ability to activate a US representation, extensive training would not affect sensitivity of CRs to reinforcer devaluation procedures.

The experiments described in this article were designed to evaluate a Pearce-Hall (1980)-based account of Holland's (1998) observation that mediated food aversion learning was obtained after small numbers of CS-food pairings but not after extensive CS-food training. These experiments replicated the amount of training effect observed by Holland (1998) and showed the effects of two manipulations designed to restore or maintain the associability of extensively trained CSs. In Experiments 1 and 2, after extensive CS-US pairings, CS associability was restored by a brief extinction phase prior to assessment of new learning. In Experiment 3, CS associability was maintained over extensive training by intermixing nonreinforced CS presentations throughout training. In both cases, violation of CS-US expectancy produced by US omission should restore or maintain CS associability relative to treatments without expectancy-violating extinction trials. In turn, this restoration of associability should enable the acquisition of a mediated flavor aversion, even after extensive CS-US training.

\section{EXPERIMENT 1}

According to the Pearce-Hall model, extensive CS-US pairings reduce the associability of the CS, making it less sensitive to new learning. Hall and Pearce (1979) found that interposing an extinction (CS-alone) trial between extensive tone-shock training and pairings of the same tone with a more intense shock enhanced learning of associations between that tone and the more intense shock. In the Pearce-Hall model, by generating a discrepancy between the expected reinforcer and the reinforcer actually received ("surprise"), shock omission on the extinction trial enhanced the associability of the tone on subsequent trials, allowing it to more rapidly accrue associative strength on the basis of the new, more intense shock. At the same time, because the tone's associability would be low on the extinction trial itself, shock omission would have little direct extinction effect on the tone's existing associative strength.

In Experiment 1, rats first received extensive training with one CS and minimal training with a second CS; both 
CSs were paired with the same food pellet reinforcer. Then, separate groups of rats received a brief extinction or surprise phase with the minimally trained CS, the extensively trained CS, or no explicit event. Next, one of the CSs was paired with a lithium chloride $(\mathrm{LiCl})$ injection in an attempt to establish an aversion to the food pellets, mediated by a CS-activated representation of food. Finally, pellet consumption was examined to assess aversion learning. If representation-mediated learning of a food aversion is reduced after extensive CS-US pairings because the associability of the CS-activated representation of food pellets is driven down (as the Pearce-Hall model suggests), then the surprise occasioned by a brief period of nonreinforcement of that CS should enhance its associability. Thus, rats that receive brief extinction of the extensively trained $\mathrm{CS}$ before that $\mathrm{CS}$ is paired with $\mathrm{LiCl}$ should show more evidence of food pellet aversion than rats that do not receive extinction trials. By contrast, the minimally trained CS is unlikely to have lost much of its associability (see, e.g., Holland, Bashaw, \& Quinn, 2002; Kaye \& Pearce, 1984), and so the surprise trials are unlikely to have much incremental effect on that CS's associability. Indeed, that treatment might reduce the minimally trained CS's ability to activate a representation of the food US, and hence might reduce the formation of a mediated food aversion relative to that of rats that do not receive nonreinforced presentations of that CS.

\section{Method}

Subjects and Apparatus. Thirty male albino rats (CD strain, Charles River Laboratories, Raleigh, NC), each weighing 300-325 g upon arrival at the vivarium, were housed individually with ad lib access to water. The rats were maintained at $85 \%$ of their baseline weights by limiting their access to food to a single daily meal. The vivarium was climate controlled and illuminated from 6 a.m. to 8 p.m.

The apparatus consisted of eight individual chambers $(22.9 \times$ $20.3 \times 20.3 \mathrm{~cm}$ ) with aluminum front and back walls, clear acrylic side walls and top, and a floor made of $0.48-\mathrm{cm}$ stainless steel rods spaced $1.9 \mathrm{~cm}$ apart. A dimly illuminated food cup was recessed in the center of the front wall. An infrared photocell placed just inside the food cup was polled $(1 \mathrm{kHz})$ by computer circuitry. Each chamber was enclosed in a sound-resistant shell. A 6-W houselight was mounted on the inside wall of the shell, $10 \mathrm{~cm}$ above the experimental chamber and even with the front wall. Ventilation fans provided masking noise $(70 \mathrm{~dB})$. Constant dim illumination was provided by a 6-W lamp behind a dense red lens mounted on the ceiling of the shell.
Procedure. Table 1 shows an outline of the training procedure that was used in Experiment 1. The rats were first trained to eat from the recessed food cup in a single 64-min session, which included 16 deliveries of the reinforcer: two 45-mg Noyes food pellets (Research Diets, New Brunswick, NJ). Next, the rats received eight 64-min training sessions to establish extensive training of one CS and minimal training of another CS. In each of these sessions, there were 14 reinforced presentations of one CS and 2 reinforced presentations of the other. For half of the rats, the minimally trained CS was a 10 -sec illumination of the light and the extensively trained CS was a $10-\mathrm{sec}, 78-\mathrm{dB}, 1500-\mathrm{Hz}$ tone. For the other half of the rats, the identities of the two CSs were reversed. Then, the rats were divided into four groups ( $n \mathrm{~s}=7$ or 8 ) and given a single 16-min session designed to increase the associability of the extensively trained CS in the extensive-surprise group prior to mediated aversion training. In this session, the rats in the extensive-surprise and minimal-surprise groups received four nonreinforced presentations of the extensively trained CS or the minimally trained CS, respectively, and the rats in the extensive-no surprise and minimal-no surprise groups received only placement in the chambers.

On the next day, the rats received $10 \mathrm{~min}$ of exposure in the experimental chambers to a white ceramic bowl that contained 100 pellets, as a pretest of the consumption test procedure. Over the next 4 days, the rats received four 5-min sessions designed to establish an aversion to the food pellet reinforcer via pairing of a presentation of one of the CSs (but not pellets) with the toxin LiCl. In the first and third of these sessions, there was a single 10 -sec presentation of one of the CSs in the absence of food. Immediately after this CS presentation, the rats were removed from the chambers and injected with $5 \mathrm{ml} / \mathrm{kg} 0.3 \mathrm{M} \mathrm{LiCl}$ and returned to their home cages. For the rats in the extensive-surprise and extensive-no surprise groups, this CS was the extensively trained CS, and for the remaining rats it was the minimally trained CS. Regardless of whether the surprise session included the extensively trained CS, and regardless of whether the toxin-paired CS was the minimally trained or the extensively trained CS, the identity of the CS (light or tone) was completely counterbalanced. In the second and fourth sessions of the mediated aversion training phase, the rats were placed in the chambers for $5 \mathrm{~min}$ but no events were delivered. Finally, on the next day, the rats received $10 \mathrm{~min}$ of exposure in the experimental chambers to a white ceramic bowl that contained 100 pellets, as a test of the effects of the mediated aversion training sessions.

\section{Results}

Figure 1 shows the acquisition of conditioned responding during the initial training phase. Conditioned food cup responding was acquired rapidly to each CS, although the tone elicited more responding than the light, especially with minimal training. As would be anticipated, responding was greater to the extensively trained CSs than to the

Table 1

Outline of Procedure of Experiment 1

\begin{tabular}{|c|c|c|c|c|c|}
\hline Group Name & Training & Surprise & Pretest & Mediated Aversion & Test \\
\hline Extensive-surprise & $\begin{array}{r}112 \mathrm{CS}_{\text {extensive }} \rightarrow \text { food } \\
16 \mathrm{CS}_{\text {minimal }} \rightarrow \text { food }\end{array}$ & $\mathrm{CS}_{\text {extensive }} \rightarrow$ nothing & food & $\mathrm{CS}_{\text {extensive }} \rightarrow \mathrm{LiCl}$ & food \\
\hline Extensive-no surprise & $\begin{aligned} 112 \mathrm{CS}_{\text {extensive }} & \rightarrow \text { food } \\
16 \mathrm{CS}_{\text {minimal }} & \rightarrow \text { food }\end{aligned}$ & chamber only & food & $\mathrm{CS}_{\text {extensive }} \rightarrow \mathrm{LiCl}$ & food \\
\hline Minimal-surprise & $\begin{aligned} 112 \mathrm{CS}_{\text {extensive }} & \rightarrow \text { food } \\
16 \mathrm{CS}_{\text {minimal }} & \rightarrow \text { food }\end{aligned}$ & $\mathrm{CS}_{\text {minimal }} \rightarrow$ nothing & food & $\mathrm{CS}_{\text {minimal }} \rightarrow \mathrm{LiCl}$ & food \\
\hline Minimal-no surprise & $\begin{array}{r}112 \mathrm{CS}_{\text {extensive }} \rightarrow \text { food } \\
16 \mathrm{CS}_{\text {minimal }} \rightarrow \text { food }\end{array}$ & chamber only & food & $\mathrm{CS}_{\text {minimal }} \rightarrow \mathrm{LiCl}$ & food \\
\hline
\end{tabular}

Note-The numbers listed in the Training column refer to the total number of trials of each type. CS, conditioned stimulus; $\mathrm{LiCl}$, lithium chloride; $\rightarrow$, followed by. 


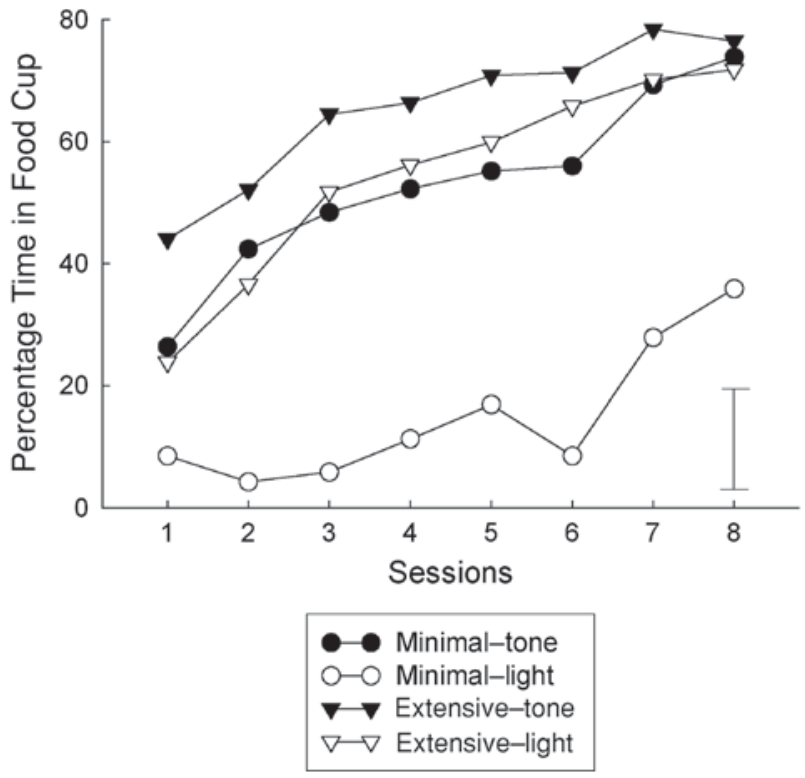

Figure 1.Acquisition of food cup responding in the initial training phase of Experiment 1. In each session, there were 2 presentations of the conditioned stimulus that received minimal training and 14 presentations of the conditioned stimulus that received extensive training. The error bar indicates twice the within-subjects mean standard error.

minimally trained CSs. Food cup responding was subjected to a five-way ANOVA with variables of cue identity (tone vs. light), subsequent mediated aversion condition assignment (minimally vs. extensively trained CS paired with $\mathrm{LiCl}$ ), subsequent surprise assignment (surpriseextinction vs. no surprise), amount of training of the CS (minimal vs. extensive), and sessions. The effects of cue identity $[F(1,22)=8.00]$, number of training trials $[F(1,22)=126.11]$, and sessions $[F(7,154)=42.53]$ were significant, as were the interactions of cue identity with number of training trials $[F(1,22)=109.61]$ and sessions $[F(7,154)=2.86]$ and the interaction of number of training trials with sessions $[F(7,154)=4.10]$. Neither of the subsequent assignment variables nor any of their interactions was significant $\left(F_{\mathrm{S}}<1.5\right)$. A similar ANOVA of baseline responding prior to each trial $(M \mathrm{~s}<10 \%)$ showed no significant effects or interactions $\left(F_{\mathrm{s}}<1.5\right)$.

Table 2 shows responding to the CSs in the surprise and mediated learning sessions. In these sessions, responding maintained the patterns it had at the end of initial training. The surprise treatment partially extinguished responding: The rats that received the surprise treatment showed less responding in the mediated aversion sessions than did those that received only context exposure in those sessions. A cue identity $\times$ amount of training ANOVA showed significant main effects of both variables $\left[F_{\mathrm{s}}(1,11)>7.66\right]$, but their interaction was not reliable $(F<1)$. A cue identity $X$ previous surprise treatment $X$ amount of training of surprise cue $X$ session ANOVA of responding on the mediated aversion trials showed significant main effects of cue identity, previous surprise treatment, and amount of training $\left[F_{\mathbf{s}}(1,22)>4.11\right]$ but not of sessions $[F(1,22)=$ 3.12]. In addition, amount of training interacted significantly with both cue identity and prior surprise treatment $\left[F_{\mathrm{s}}(1,22)>4.82\right]$; cue identity mattered more after few training trials, and the surprise trials produced greater loss after extensive training. An ANOVA of pre-CS responding showed no effects $\left(F_{\mathrm{s}}<1\right)$.

Figure 2 shows the primary data from this experimentnamely, the consumption of food pellets from the ceramic bowls in the experimental chambers. The rats that received pairings of the minimally trained CS with toxin consumed fewer pellets than did the rats that received the extensively trained CS paired with toxin. This outcome is consistent with the claim that the rats acquired a stronger aversion to the food when a minimally trained CS was paired with toxin than when an extensively trained CS was paired with toxin, as Holland (1998) noted.

Critically, the surprise manipulation had no effect on mediated aversion learning. The rats that received toxin after the minimally trained CS showed less consumption in the test session (right bars in Figure 2A) and more reduction of consumption (Figure 2B) from the pretest levels than did the rats that were made ill after the extensively trained CS. There were no differences among the groups in consumption during the pretest (left bars in panel A).

A cue identity $X$ surprise $X$ amount of training of the toxin-paired CS ANOVA of pretest consumption showed no significant effects $(F \mathrm{~s}<1)$ except for the cue identity $\times$ amount of training interaction $[F(1,22)=$ $2.89, p>.10]$. A comparable ANOVA of test responding showed a reliable effect of amount of training of the toxin-paired $\mathrm{CS}[F(1,22)=8.10$; remaining $F \mathrm{~S}<1]$ except for the cue identity $\times$ amount of training interaction $[F(1,22)=3.30, p=.08]$. Finally, a comparable ANOVA

Table 2

Responding in the Surprise and Mediated Aversion Sessions of Experiment 1

\begin{tabular}{|c|c|c|c|c|c|c|}
\hline \multirow[b]{3}{*}{ Group } & \multirow{2}{*}{\multicolumn{2}{|c|}{$\begin{array}{c}\text { Surprise } \\
\text { (Trials 1-4) }\end{array}$}} & \multicolumn{4}{|c|}{ Mediated Aversion } \\
\hline & & & \multicolumn{2}{|c|}{ Trial 1} & \multicolumn{2}{|c|}{ Trial 2} \\
\hline & $M$ & $S E$ & $M$ & $S E$ & $M$ & $S E$ \\
\hline \multicolumn{7}{|c|}{ Extensive-surprise } \\
\hline Tone & 65.2 & 1.3 & 33.2 & 14.0 & 10.1 & 11.6 \\
\hline Light & 48.7 & 5.4 & 31.9 & 5.2 & 25.2 & 14.5 \\
\hline Pre-CS & 4.8 & 3.2 & 4.1 & 1.9 & 3.8 & 3.1 \\
\hline \multicolumn{7}{|c|}{ Extensive-no surprise } \\
\hline Tone & - & & 73.4 & 14.0 & 60.9 & 11.6 \\
\hline Light & - & & 68.9 & 6.7 & 39.6 & 15.4 \\
\hline Pre-CS & - & & 5.0 & 2.3 & 4.0 & 2.1 \\
\hline \multicolumn{7}{|c|}{ Minimal-surprise } \\
\hline Tone & 45.7 & 16.8 & 21.1 & 15.4 & 20.6 & 14.7 \\
\hline Light & 17.0 & 12.0 & 2.7 & 2.1 & 1.6 & 1.8 \\
\hline Pre-CS & 3.5 & 1.6 & 4.1 & 2.0 & 2.8 & 1.9 \\
\hline \multicolumn{7}{|c|}{ Minimal-no surprise } \\
\hline Tone & - & & 47.5 & 14.2 & 31.1 & 20.5 \\
\hline Light & - & & 15.3 & 12.0 & 18.2 & 8.8 \\
\hline Pre-CS & - & & 3.9 & 1.9 & 2.9 & 1.6 \\
\hline
\end{tabular}

Note-Entries are for mean $(M)$ percentage time in food cup and standard error $(S E)$. 


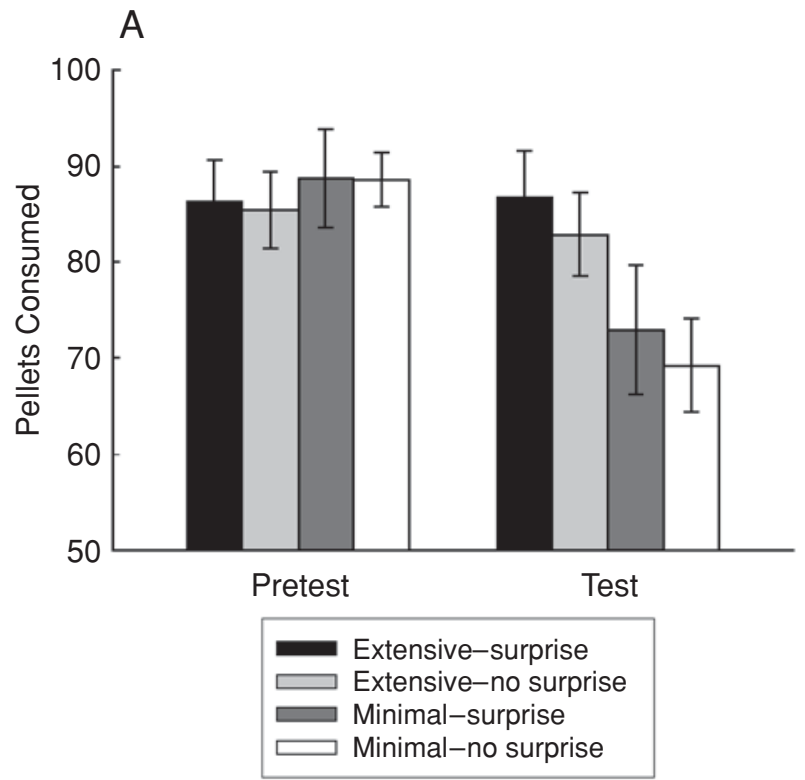

B

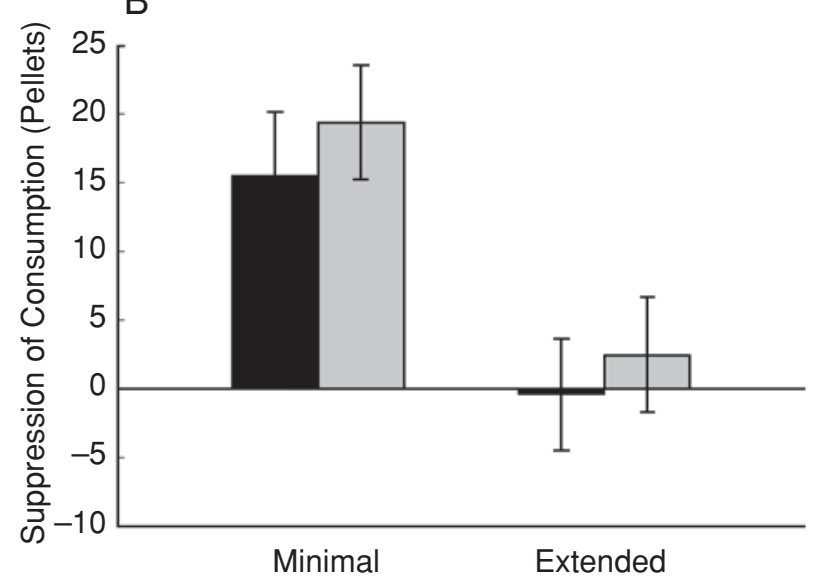

Surprise

No surprise

Figure 2. Mediated food aversion learning in Experiment 1. Panel A shows mean $( \pm S E M)$ food consumption in the pretest and test phases, and panel $B$ shows the mean $( \pm S E M)$ change in consumption between those tests (pretest score minus test score). In panel $B$, reductions in consumption (positive change scores) reflect the acquisition of a food aversion.

that also included test versus pretest as a variable showed significant effects of that variable $[F(1,22)=20.72]$ and of its interaction with the amount of training of the toxinpaired CS $[F(1,22)=16.17]$. No other effect or interaction approached significance except for the cue identity $\times$ amount of training interaction $[F(1,22)=3.93, p=.06]$.

\section{Discussion}

The results of Experiment 1 replicated those of Holland (1998): Food consumption was reduced after pairings of a minimally trained $\mathrm{CS}$ with $\mathrm{LiCl}$ but not after pairings of an extensively trained CS with $\mathrm{LiCl}$. Although, unlike the previous experiments, Experiment 1 did not include nonassociative controls (e.g., toxin presentations unpaired with any CS-a control provided in Experiment 3), it seems reasonable to infer from these data that acquisition of a food aversion, mediated by a CS-activated representation of food, was greater after minimal than after extensive training. Notably, CS extinction trials administered prior to $\mathrm{CS}-\mathrm{LiCl}$ pairings, intended to restore the associability of the food representation activated by the extensively trained CS, were not effective in altering the acquisition of the mediated taste aversion after either minimal or extensive training. Thus, there was no evidence that losses in the associability of a CS-activated representation of food pellets with extensive CS-US training were critically involved in the loss of susceptibility to mediated aversion learning.

Regardless of the role of associability changes, the observation of differential effects of CS-toxin pairings on mediated aversion learning after minimal and extensive CS-food training in this procedure demands that the food representations activated by the two CSs be distinguished. Each rat received both extensive training with one CS and minimal training with another CS, each paired with the same food reinforcer. If both CSs activated the same food representation, then it should not have mattered which CS was paired with toxin. However, a food aversion was formed only if the minimally trained CS was paired with toxin.

Finally, it is notable that the brief extinction phase (four trials) substantially reduced CRs to the extinguished CSs (Table 2). In the case of the minimally trained CS, this observation indicates that even proportionally large losses in CRs to the CS had no observable effect on that CS's ability to activate a representation of food pellets for mediated learning of a taste aversion. This effect is reminiscent of Rescorla's (1996a, 1996b) observations that Pavlovian CSs apparently still controlled specific outcome expectancies after extensive extinction of CRs and even after counterconditioning with new USs. Furthermore, this observation reduces concerns that with the extensively trained CS, detrimental effects of extinction on the ability of the CS to activate a food representation may have offset any advantageous effects of the surprise treatment on the associability of the CS-activated food representation.

\section{EXPERIMENT 2}

The logic of Experiment 1 demands that the surprise procedure restore the associability of the extensively trained CS. The absence of any enhancement of mediated aversion learning by surprise in Experiment 1 may simply reflect ineffectiveness of that procedure in restoring associability, rather than a lack of an effect of such a restoration. The question addressed in Experiment 2 was whether or not the surprise procedures of Experiment 1 are sufficient to restore CS associability, as measured by simple acquisition of new learning to the extensively trained CS itself. The acquisition and surprise procedures 
were identical to those used in Experiment 1. However, the mediated aversion phase was replaced by pairings of the test CS with a new, higher magnitude (five pellets rather than two) and a qualitatively preferred reinforcer (fruitpunch-flavored sucrose rather than grain). If the surprise procedure of Experiment 1 restored putative losses in the associability of the extensively trained $\mathrm{CS}$, then rats that received that treatment should show more rapid acquisition of conditioned responding appropriate to the new reinforcer. For half of the rats, the new reinforcer was delivered to the same food cup as the original reinforcer, and for the other half it was delivered to a previously unused cup on the opposite side of the chamber. The purpose of using two different delivery sites for some of the rats in the test phase was to evaluate any role of summation of, or competition between, the peripheral CRs established with the old and new reinforcers. Because the primary concern of the present study is why extensive training reduces mediated aversion learning, in Experiment 2 only the extensively trained CS served as a target cue.

\section{Method}

Subjects and Apparatus. The subjects were 24 male albino rats obtained and maintained as in Experiment 1. The apparatus included six of the chambers used in Experiment 1; in addition, a second recessed food cup, located on the wall of the chamber opposite that of the original cup, was made available in the test phase for half of the rats.

Procedure. Table 3 shows an outline of the procedure of Experiment 2 . The training and surprise sessions were identical to those received by the rats in the extensive-surprise and extensive-no surprise groups of Experiment 1. However, instead of mediated aversion training, these rats received two 64-min sessions, each with eight pairings of the extensively trained CS with a new reinforcer, five fruit-punch-flavored sucrose pellets (Research Diets), to assess the surprise session's ability to enhance the associability of that CS. For half of the rats in each training and counterbalancing condition, the new reinforcer was delivered to the same food cup as the other pellets, but for the other half of the rats it was delivered to a similar food cup on the opposite wall of the chamber. For the latter rats, the opening of the original food cup was blocked in this test.

\section{Results}

As in Experiment 1, conditioned food cup responding was acquired rapidly to each CS, although the tone elicited more responding than the light early in training. In the final training session, the percentages of time in the food cup during the extensively trained light and tone stimuli were $77.1 \% \pm 3.8 \%$ and $76.4 \% \pm 4.1 \%$, respectively; to the minimally trained light and tone, they were $42.1 \% \pm$ $6.5 \%$ and $77.4 \% \pm 5.1 \%$, respectively. Pre-CS responding was less than $10 \%$ and did not differ across groups $(F \mathrm{~s}<$ 1). Food cup responding during the CSs was subjected to a three-way ANOVA, with variables of cue identity (tone vs. light), subsequent surprise assignment (surprise vs. no surprise), and amount of training of the CS (minimal vs. extensive). The main effects of cue identity $[F(1,20)=8.27]$ and amount of training $[F(1,20)=34.92]$ and the interaction of cue identity with amount of training $[F(1,20)=$ $36.06]$ were all significant; the effects of subsequent assignment to surprise condition were not $(F<1)$.

As in Experiment 1, responding in the surprise session maintained the pattern seen at the end of initial training. Responding to the extensively trained light and tone was $48.0 \% \pm 7.0 \%$ and $53.0 \% \pm 6.9 \%$, respectively, which did not differ significantly $(F<1)$. Pre-CS responding was $3.6 \% \pm 3.1 \%$.

Figure 3 shows the primary data of Experiment 2-that is, the acquisition of CRs to the extensively trained CS as it was paired with a new reinforcer. In all conditions, responding rapidly rose to a new, higher asymptote. However, in the first test session the rats that had received nonreinforced surprise presentations of the extensively trained CS prior to test pairings with the new US showed more responding than did the rats that did not receive those presentations, despite starting that session (Trial 1) with lower levels of responding to the CS. This superiority in acquisition was observed regardless of whether the new reinforcer was delivered to the original food cup location or to a new location.

Separate CS identity $\times$ surprise condition $\times$ trial ANOVAs for responding in the first test session were conducted for the rats that received the new reinforcer in the old location and those that received it in the new location. For both locations, there were significant main effects of surprise $[F \mathrm{~s}(1,8)>6.16]$ and trial $[F \mathrm{~s}(7,56)>13.13]$ and significant surprise $\times$ trial interactions $\left[F_{\mathbf{s}}(7,56)>\right.$ 11.94]. Separate analyses for the first trial only also showed significant main effects of surprise $[F \mathrm{~s}(1,8)>5.29]$; note that the differences on Trial 1 were in the direction opposite that of the differences in the remaining sessions. This

Table 3

Outline of Procedure of Experiment 2

\begin{tabular}{lrll}
\hline Group Name & \multicolumn{1}{c}{ Training } & Surprise & Test \\
\hline Surprise & $112 \mathrm{CS}_{\text {extensive }} \rightarrow$ food & $\mathrm{CS}_{\text {extensive }} \rightarrow$ nothing & $\mathrm{CS}_{\text {extensive }} \rightarrow$ new food \\
& $16 \mathrm{CS}_{\text {minimal }} \rightarrow$ food & & \\
No surprise & $112 \mathrm{CS}_{\text {extensive }} \rightarrow$ food & chamber only & $\mathrm{CS}_{\text {extensive }} \rightarrow$ new food \\
& $16 \mathrm{CS}_{\text {minimal }} \rightarrow$ food & & \\
\hline
\end{tabular}

Note-The numbers listed in the Training column refer to the total number of trials of each type. The new food was delivered in the original food cup to half of the rats in each group, and in a new food cup to the other half of the rats. CS, conditioned stimulus; $\mathrm{LiCl}$, lithium chloride; $\rightarrow$, followed by. 


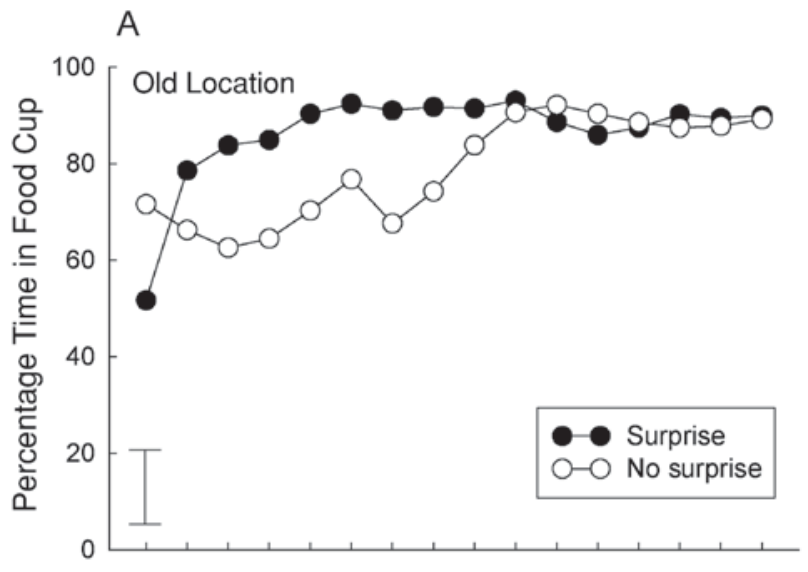

B

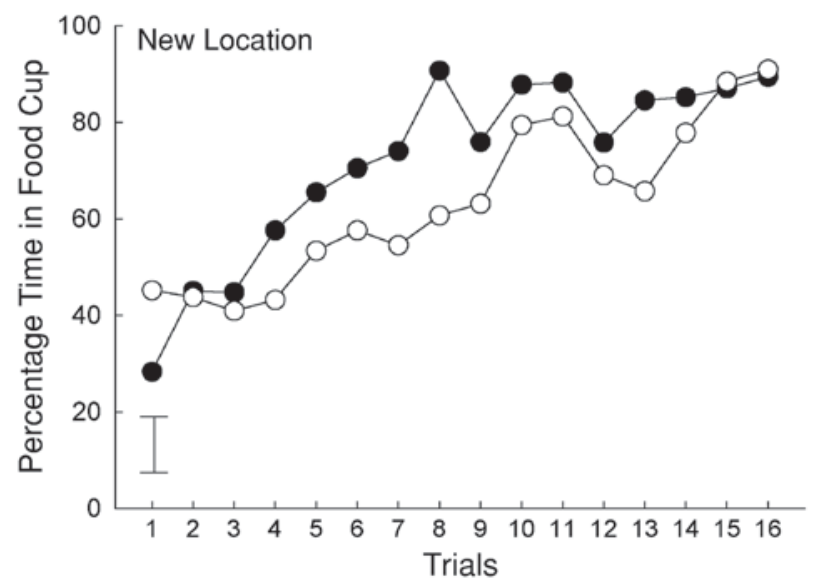

Figure 3. Acquisition of new learning in the test phase of Experiment 2. The error bars show twice the between-groups mean standard error.

difference in responding on Trial 1 reflects extinction of responding in the rats that received surprise (extinction) trials with the CS prior to testing.

Pre-CS responding was below $10 \%$ in all conditions and did not differ across groups $(F \mathrm{~s}<1)$.

\section{Discussion}

The results of Experiment 2 extend the findings of Hall and Pearce (1979) to an appetitive conditioning preparation. Although the brief extinction treatment used to induce surprise produced significant losses in the ability of CSs to elicit already established CRs, it enhanced the rate of new learning about those CSs. That enhancement occurred regardless of whether the indicator of new learning was the same as or different from the CR previously controlled by the CS (entry into the same or a different food cup).

These results provide evidence that the extensive training used in Experiments 1 and 2 indeed reduced CS associability, and that the surprise procedures used in these experiments are effective in restoring the associability of the extensively trained CS. If the failure of CS-toxin pair- ings to produce a mediated food aversion after extensive CS training in Experiment 1 was due to losses in CS associability produced by that extensive training, then the surprise manipulation in that experiment should have restored CS associability and enabled mediated food aversion learning. The lack of such an effect of surprise on mediated aversion learning in Experiment 1 suggests that the inability of the extensively trained CS to establish a mediated taste aversion in Experiment 1 was not due to losses in its associability.

\section{EXPERIMENT 3}

In the Pearce-Hall model, CS associability on any trial is determined by the accuracy with which its consequences were predicted on previous trials. Thus, the associability of a CS should be maintained at a higher value with partial reinforcement procedures than with consistent reinforcement procedures. Using orienting responses as an indicator of associability, Pearce and colleagues (e.g., Collins \& Pearce, 1985; Collins, Young, Davies, \& Pearce, 1983; Kaye \& Pearce, 1984; Pearce, Kaye, \& Collins, 1985) found substantially greater associability of CS trained with a variety of partial reinforcement procedures than of that trained with consistent reinforcement procedures.

In Experiment 3, the acquisition of a mediated taste aversion after CS-illness pairings was examined after different amounts of training of CSs with either partial or consistent reinforcement procedures. In contrast to Experiment 1, in Experiment 3 the effects of amount of training were evaluated between groups. Each rat received training with two CSs, one consistently paired with food and the other paired with food on $50 \%$ of its presentations. Different groups of rats received 20,40 , or 80 training trials. If the amount of training effect is due to associability losses over extensive training, and if partial reinforcement maintains CS associability better than consistent reinforcement, then, as the number of training trials increases, the rats that receive pairings of the partially reinforced CS with toxin should acquire stronger mediated food aversions than those that receive pairings of the consistently reinforced $\mathrm{CS}$ with toxin. Although some investigators have reported difficulty in observing another consequence of partial reinforcement - that is, the partial reinforcement extinction effect - in within-subjects procedures such as these (see, e.g., Pearce, Redhead, \& Aydin, 1997; but see Rescorla, 1999), it is notable that several of Pearce's demonstrations of greater orienting to partially reinforced CSs than to consistently reinforced CSs involved within-subjects comparisons.

After assessment of the mediated taste aversion, the same rats were tested in a reinforcer devaluation procedure in which the food itself was paired (for some rats) with toxin, and then responding to the CSs was assessed. This test permitted the comparison, in the same rats, of the effects of amount of training and partial reinforcement on performance on the devaluation task with those effects on mediated aversion learning. Recall that Holland (1998, Experiment 3) found that devaluation task performance was insensitive to 
variations in the amount of training that had critical effects on the observation of mediated aversion learning.

\section{Method}

Subjects and Apparatus. The subjects were 60 male albino rats obtained and maintained as in Experiments 1 and 2. The apparatus was the same as that used in Experiment 1.

Procedure. Table 4 shows an outline of the procedure of Experiment 3, which was conducted in two similar replications, one with 28 rats and one with 32 rats. All of the rats first received a food cup training session, as in Experiments 1 and 2. Then, they were separated into three groups, which received different numbers of consistent reinforcement (CRF) and partial reinforcement (PRF) training. The rats in Group 2040 received 20 reinforced presentations of one (CRF) CS, and 20 reinforced and 20 nonreinforced presentations of another (PRF) CS. The rats in Group 4080 received 40 reinforced presentations of the CRF CS, and 40 reinforced and 40 nonreinforced presentations of the PRF CS. The rats in Group 8080 received 80 reinforced presentations of the CRF CS, and 40 reinforced and 40 nonreinforced presentations of the PRF CS. Therefore, in Group 8080 the number of PRF CS and CRF CS presentations (80) was equated, whereas in Groups 2040 and 4080 the number of reinforced presentations was equated (20 and 40, respectively) for the CRF CS and the PRF CS; likewise, the number of CRF CS presentations in Group 4080 and the number of PRF CS presentations in Group 2040 (40) were equated. Each training session included four reinforced and four nonreinforced PRF CS presentations and either four (Groups 2040 and 4080) or eight (Group 8080) reinforced CRF CS presentations. The intertrial intervals $(M=4 \mathrm{~min}$; range, $2-6 \mathrm{~min})$ were equated across all groups. Thus, the rats in Group 8080 received ten 64-min training sessions, the rats in Group 4080 received ten 48min training sessions, and the rats in Group 2040 received five 48-min training sessions. In each group, the identities of the CRF and PRF CSs (the 10-sec light and tone CSs used in the previous studies) were counterbalanced.

After a 10-min pretest of consumption of food pellets in the experimental chamber, as in Experiment 1, the rats received mediated aversion training identical to that of Experiment 1 except that the rats rested in their home cages (rather than in the experimental chambers) on the 2 nd and 4 th days. For 6-8 of the rats in each group, the CRF CS was paired with $\mathrm{LiCl}$, for 6-8 rats the PRF CS was paired with $\mathrm{LiCl}$, and for 6 rats no $\mathrm{CS}$ was presented prior to $\mathrm{LiCl}$ injections. The identities of these CSs (light or tone) were counterbalanced as closely as possible. On the next day, a consumption test, identical to that used in the pretest session, was conducted.

Finally, a standard reinforcer devaluation procedure was administered over 5 days. On Days 1 and 3, half of the rats (counterbalanced as much as possible across all previous training and testing conditions) received $10 \mathrm{~min}$ of access to the ceramic bowls with 100 pellets in their home cages, followed immediately by $\mathrm{LiCl}$ injection (paired condition); the other half received only the $\mathrm{LiCl}$ injections (unpaired condition). On Days 2 and 4, the rats in the unpaired condition received $10 \mathrm{~min}$ of access to the bowls of pellets but no injections. On the last day of the experiment, all of the rats received a 32-min test session to assess the effects of flavor aversion training on food cup responding to the two CSs. This session included four nonreinforced presentations of each of the two CSs, randomly intermixed.

\section{Results}

Rats in all of the groups rapidly acquired CRs to both CSs in the initial training phase. As in Experiments 1 and 2 , the tone elicited more responding than the light, especially with small amounts of training. Partially and consistently reinforced CSs controlled similar levels of con-

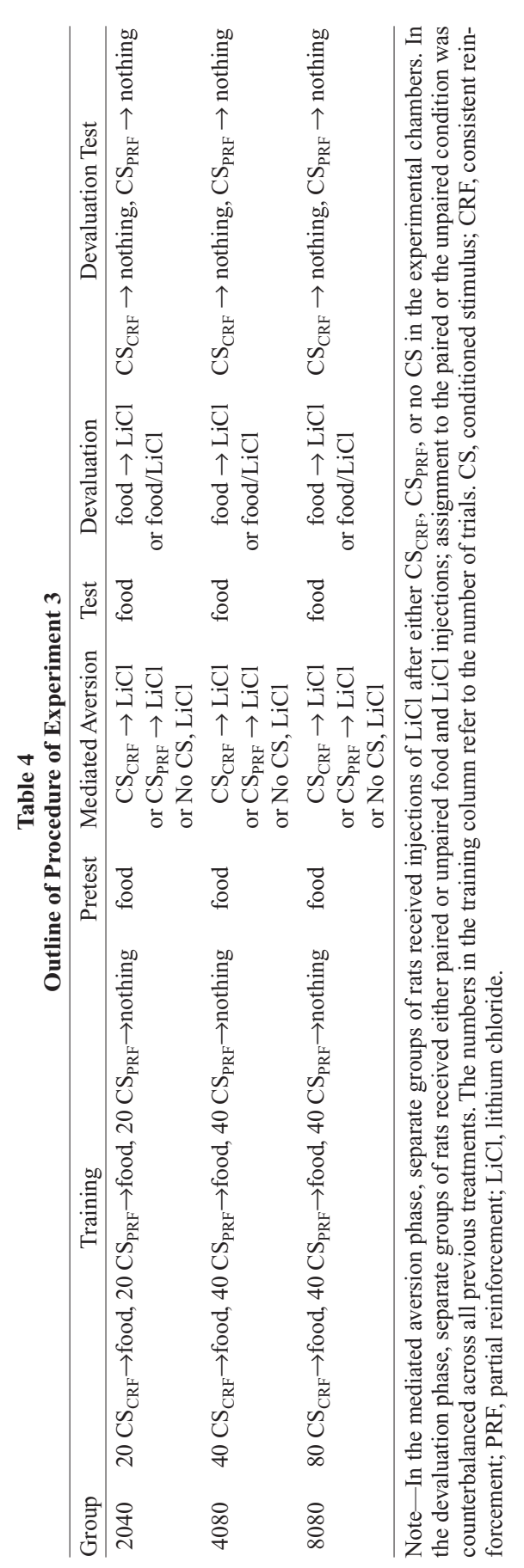




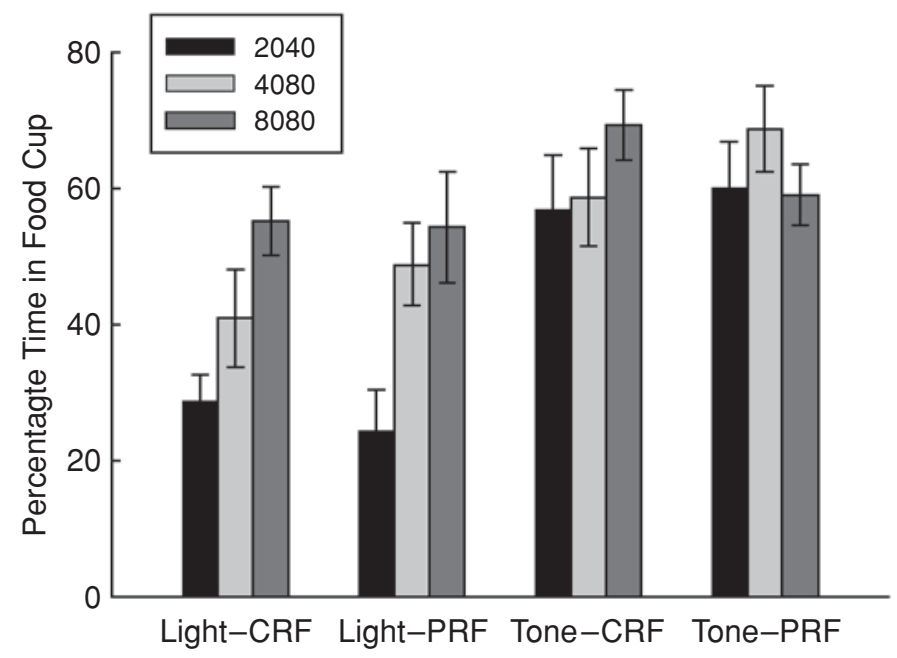

Figure 4. Mean $( \pm S E M)$ food cup responding during the tone and light conditioned stimuli in the last session of initial training in Experiment 3. The first two digits of each group name indicate the total number of CRF trials, and the second two digits indicate the total number of PRF trials received in that phase. $\mathrm{CRF}$, consistently reinforced conditioned stimulus; PRF, partially reinforced conditioned stimulus.

ditioned responding; notably, except in Group 8080, the number of reinforced trials for the PRF and CRF CSs were matched. Figure 4 shows responding to the CSs in the final conditioning session. A group (2040 vs. 4080 vs. 8080$) \times$ cue identity (tone vs. light) $\times$ contingency $(\mathrm{CRF}$ vs. PRF) ANOVA showed significant effects of group $[F(2,54)=$ 5.66] and cue identity $[F(1,54)=69.31]$ and a significant interaction of group and cue identity $[F(2,54)=$ 7.32]. With the light CS, there was a significant effect of group for both CRF and PRF cues $\left[F_{\mathrm{S}}(1,54)>10.49\right]$, but with the tone CS those effects were not significant $(F<$ $2.11)$. Values of pre-CS responding in the final session were $5.1 \% \pm 2.1 \%, 5.3 \% \pm 2.9 \%$, and $4.9 \% \pm 2.0 \%$ for Groups 2040, 4080, and 8080, respectively $(F \mathrm{~s}<1)$.

Figure 5 shows the primary data of Experiment 3namely, the consumption of food pellets before (pretest, panel A) and after (test, panel B) mediated aversion training. As in the previous experiments, only the rats that received minimal training of a CS (Group 2040) showed evidence of mediated aversion learning in the test session. The rats in Group 2040 that received CS-toxin pairings ate fewer pellets than did the rats that received toxin without CS presentations. Furthermore, the rats that received the pairings showed less consumption in the test session than did the rats that were made ill after more extensive CS-food training. Although the Group 2040 subjects that received the pairings also consumed fewer pellets in the pretest than did those in the other groups (panel A), perhaps because they had the least prior exposure to pellets, they showed greater reduction of consumption from that pretest baseline than did the other rats (panel C). Finally, there was no evidence that the rats made ill after the partially reinforced CSs showed greater mediated aversion learning than did those made ill after the consistently reinforced CS; indeed, the nonsignificant trend was in the opposite direction.

A group $\times$ cue identity (tone vs. light trained as CRF cue) $\times$ aversion cue (CRF CS vs. PRF CS vs. no CS) ANOVA of test consumption (Figure 5B) showed significant effects of group $[F(2,42)=6.55]$ and aversion cue $[F(2,51)=4.69]$ but not of the counterbalancing variable (cue identity; $F<1$ ). An analysis that contrasted consumption after pairings of the CRF CS against consumption after pairings of the PRF CS with toxin, and which excluded the rats made ill in the absence of any CS, approached significance across all three groups $[F(1,42)=$ $3.75, p=.06]$ but did not approach significance in any of the individual groups $(p \mathrm{~s}>.10)$. Recall, however, that this nonsignificant trend was in the direction opposite that predicted by the hypothesis under investigation. Most important, a group $\times$ aversion cue analysis, which contrasted consumption in all rats that received aversion training with a CS (CRF and PRF, pooled) against consumption of rats that received aversion training with no CS, showed a significant main effect of aversion cue $[F(1,54)=5.78]$ and a significant group $\times$ aversion cue interaction $[F(2,54)=3.24]$. Among individual groups, the simple effect of aversion cue (CS vs. no CS), which indexed mediated learning, was significant only in Group $2040[F(1,15)=32.87$; in other groups, $F \mathbf{s}<1]$.

A group $\times$ cue identity $\times$ aversion cue ANOVA of pretest consumption showed a significant effect of group $[F(2,42)=5.21]$. A post hoc Newman-Keuls analysis showed that Group 2040 consumed fewer pellets than either of the other two groups. Finally, a comparable ANOVA that also included test versus pretest as a variable 


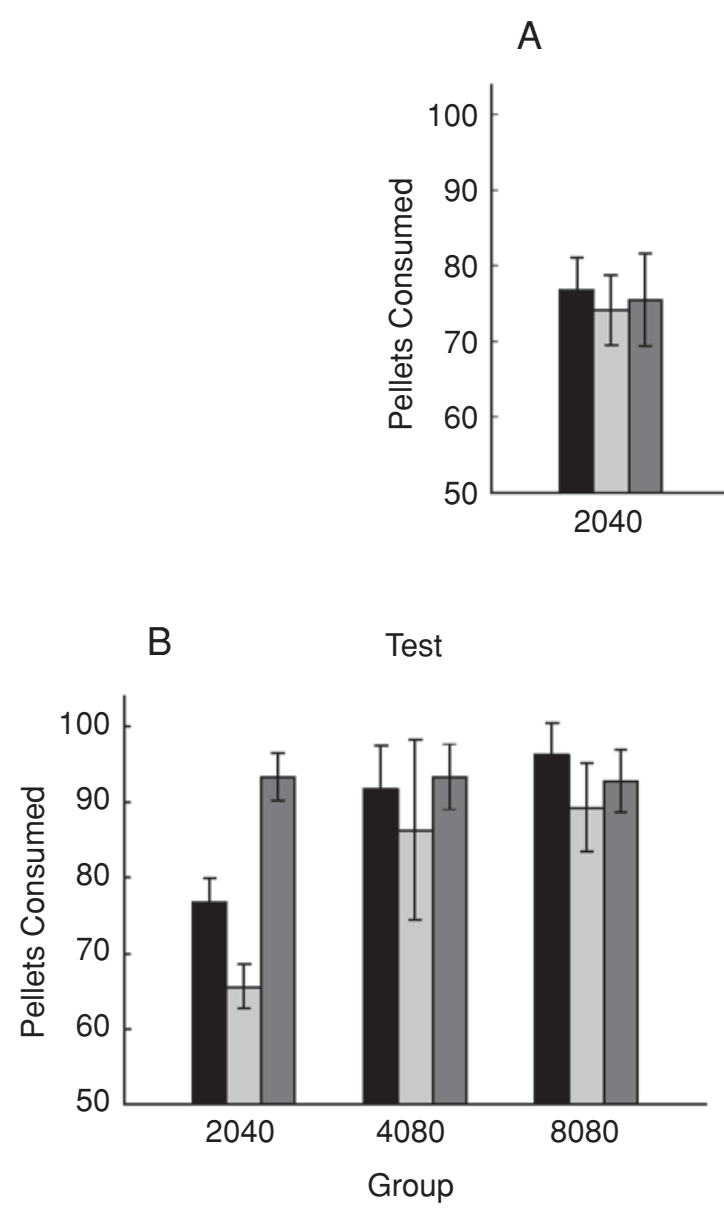

Pretest
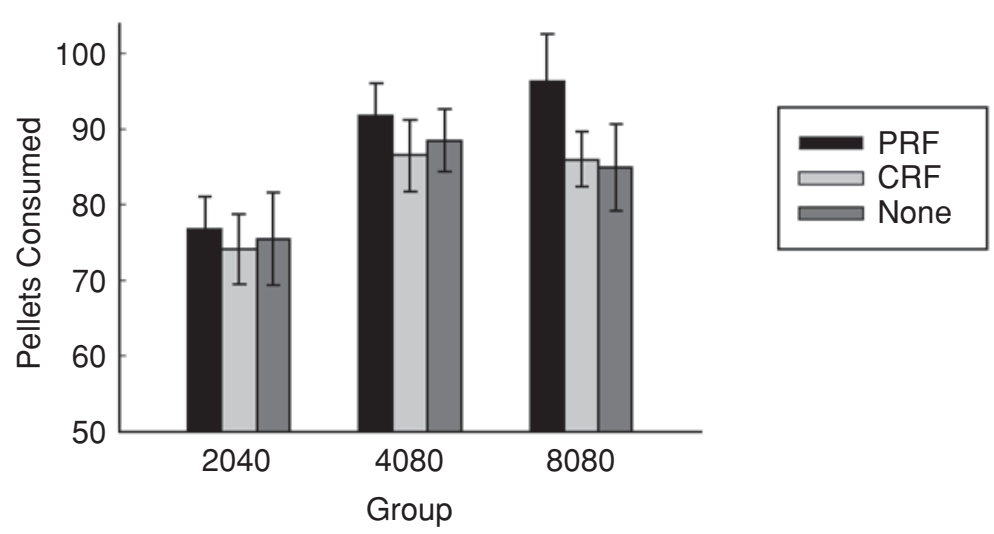

Group

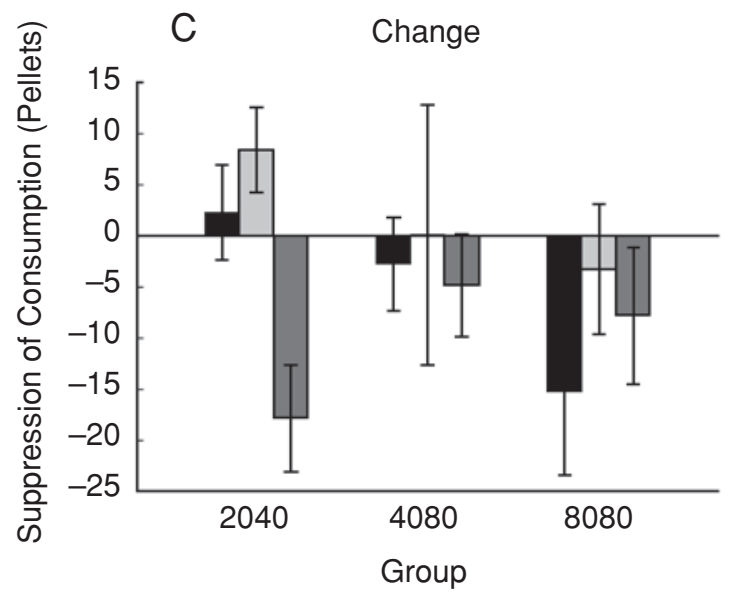

Figure 5. Mediated food aversion learning in Experiment 3. Panels A and B show mean $( \pm S E M)$ food consumption in the pretest and test sessions, respectively, and panel $\mathrm{C}$ shows the mean $( \pm S E M)$ change in consumption (pretest score minus test score). In panel $\mathrm{C}$, reductions in consumption (positive change scores) reflect the acquisition of a food aversion. The bars indicate consumption of separate subgroups of rats that received pairings of either the consistently reinforced (CRF), the partially reinforced (PRF), or no (none) conditioned stimulus with toxin between the pretest and test phases. The first two digits of each group name indicate the total number of CRF trials, and the second two digits indicate the total number of PRF trials received in the corresponding phase.

(thus evaluating the change in consumption between pretest and test) showed a significant effect of that variable $[F(1,42)=5.04]$ as well as an effect of group $[F(2,42)=$ $8.80]$. As with the test consumption scores, an overall contrast between the changes in consumption after pairings of the CRF cues and those after pairings of the PRF cues with toxin, which excluded the rats made ill in the absence of CS presentations, did not approach significance $[F(1,42)=2.05]$. Also as with the test consumption scores, a contrast used to evaluate the change in consumption between pretest and test in rats that received either the CRF or the PRF CS versus no CS was significant only in Group $2040[F(1,51)=10.16]$.

Figure 6 shows the results of the test of responding after explicit devaluation of the food reinforcer, in terms of change in responding from the final conditioning session (shown in Figure 4). The rats that had received food- $\mathrm{LiCl}$ pairings prior to testing showed less responding in testing (not shown) and more loss in responding (Figure 6) than did those that received food and $\mathrm{LiCl}$ unpaired. Although test responding was greater in groups that had received more training, neither the amount of training nor the reinforcer contingency affected the magnitude of the devaluation effect. Group (amount of training) $\times$ cue identity $\times$ devaluation contingency (paired vs. unpaired) ANOVAs of the change scores (last training session score minus test score) showed significant effects of group $[F(2,48)=$ $3.76]$, devaluation $[F(1,48)=38.24]$, and cue identity $[F(1,48)=13.82]$. No other effects or interactions were significant; notably, none of the interactions with the devaluation variable approached significance $\left(F_{\mathrm{S}}<1\right)$. A comparable ANOVA of performance on the test session itself (not shown) led to identical conclusions. Finally, neither pre-CS responding during the test session $(2.6 \%$ 


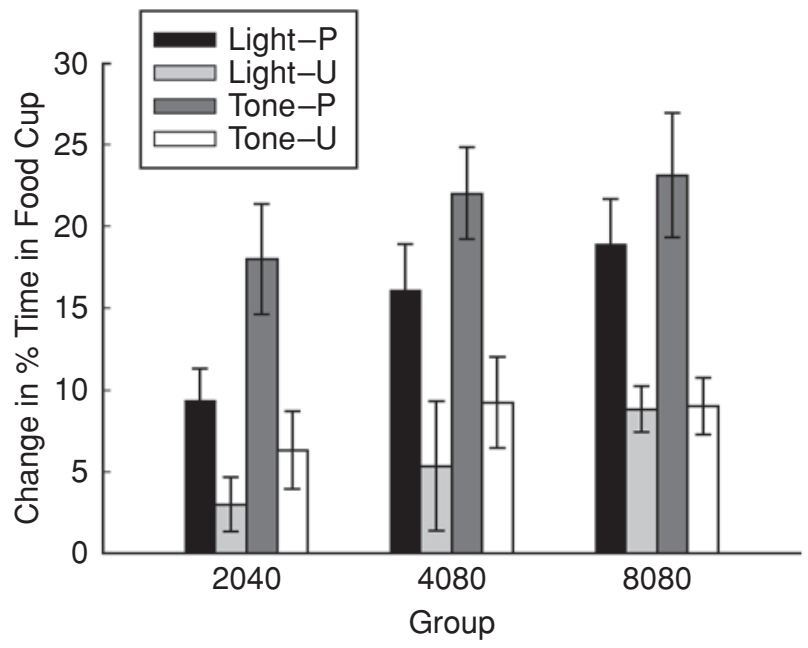

Figure 6. Results of the reinforcer devaluation test in Experiment 3. The bars indicate the mean $( \pm S E M)$ change in food cup responding to the light and tone conditioned stimuli between the final session of training and the test session. Greater reductions in responding (shown by greater positive change scores) reflect greater devaluation effects. The bars labeled $P$ show change in responding after food-toxin pairings, and the bars labeled $U$ show change in responding after unpaired presentations of food and toxin. The first two digits of each group name indicate the total number of CRF trials, and the second two digits indicate the total number of PRF trials received in the corresponding phase.

$\pm 2.1 \%, 2.4 \% \pm 1.9 \%$, and $2.6 \% \pm 1.3 \%$ in Groups 2040 , 4080 , and 8080 , respectively) nor change in pre-CS responding differed across groups $\left(F_{\mathrm{S}}<1\right)$.

\section{Discussion}

As in previous studies, mediated food aversion learning was observed when CS-illness pairings were administered after minimal numbers of CS-US pairings but not after more extensive training. Furthermore, there was no evidence that the use of a partial reinforcement contingency, which should have maintained CS associability, maintained the ability of CSs to generate mediated taste aversion learning with more extensive training. Thus, Experiment 3, like Experiment 1, provided no support for the possibility that extensive $\mathrm{CS}$-food training prevents subsequent mediated aversion learning by reducing the associability of the CS or its associatively activated food representation.

Nevertheless, CRs elicited by the CSs remained sensitive to devaluation of the food US by direct food-toxin pairings regardless of the amount of training or reinforcement contingency. This outcome supports the claim that mediated aversion learning and reinforcer devaluation performance are subserved by different aspects of associatively activated event representations.

\section{GENERAL DISCUSSION}

The results of Experiments 1 and 3 replicated those of previous studies (e.g., Holland, 1981, 1990, 1998) in show- ing that a food aversion may be established by pairing an associatively activated representation of that food (in the absence of the food itself) with illness. These results join others in showing that CS-activated food representations may substitute for food itself in a variety of functions, including the generation of CRs (Holland \& Straub, 1979), setting the occasion for responding to other CSs (Holland $\&$ Forbes, 1982a), the extinction of previously established food aversions (Holland \& Forbes, 1982b), and overshadowing or potentiating conditioning of aversions to other stimuli (Holland, 1983).

Furthermore, these results extended Holland's (1998) observation that this representation-mediated learning occurs only after relatively small amounts of training of the CS-food relation. This outcome was obtained with both between-groups (Experiment 3 ) and within-subjects (Experiment 1) designs, so it was not simply the extensive presentation of food (see, e.g., Adams, 1982) or repeated activation of a food representation that rendered that representation less susceptible to serving as a substitute for food in aversion learning. In Experiments 1 and 3, the same food was used as the reinforcer for both the minimally and the extensively trained CSs. Thus, the differential susceptibility to mediated aversion learning is apparently related to experience with a CS and its particular activation of a food representation.

The primary new conclusion from these studies is that the reduced susceptibility to mediated learning with extensive training is not due to general losses of associability of the CS or the food representation it activates, which may occur according to the Pearce-Hall (1980) model. Two manipulations designed to forestall such losses - the insertion of CS extinction trials after extensive CS-food training but before mediated aversion training (Experiment 1), and the use of partial reinforcement procedures during CS-US training (Experiment 3) — did not facilitate mediated aversion learning.

At the same time, consistent with the predictions of the Pearce-Hall (1980) model, the results of Experiment 2 demonstrated that the insertion of CS extinction trials indeed enhanced or restored CS associability after extensive training of that CS. Although the introduction of a brief CS-extinction phase after extensive CS-food training significantly reduced conditioned responding, it enhanced the subsequent acquisition of new learning to that CS when it was paired with a new food reinforcer. Thus, the results of Experiment 2 extended the findings of Hall and Pearce (1979) to an appetitive conditioning procedure. Furthermore, because the training and surprise (extinction) procedures used in Experiments 1 and 2 were identical, this outcome shows that the procedure used in Experiment 1 to restore CS associability was indeed effective. Thus, the lack of any facilitatory effect of that manipulation on mediated aversion learning in Experiment 1 implies that losses in CS associability were not responsible for the resistance to mediated aversion learning after extensive CS-food training observed in that study.

Of course, it could be argued that despite the effectiveness of this surprise procedure in restoring the associabil- 
ity of the CS itself in Experiment 2, it is insufficient to restore the associability of the CS-activated food representation. Likewise, it is possible that surprise effects of this sort are reinforcer specific. In Experiment 2, both the omitted surprising event and the reinforcer for new learning were foods, whereas in Experiment 1 the reinforcer for new learning was a toxin. Although it may seem arbitrary to assume that losses in CS associability are reinforcer general whereas recovery from those losses are reinforcer specific, there is precedent for these assumptions in the literature of unblocking with downshifts in the number of reinforcers delivered (Dickinson \& Mackintosh, 1979).

All in all, the results of the present study indicate that the ability of briefly trained CSs to mediate taste aversion learning to their reinforcers is not the result of greater associability of those CSs. However, beyond the rejection of this hypothesis, these experiments shed relatively little light on the origins of this amount of training effect. Nevertheless, it is instructive to consider alternative accounts. One such account is based on changes in processing of the US rather than on changes in that of the CS. Although the same physical food event followed all the CSs in these experiments, it may have been processed or represented differently when delivered after briefly trained CSs than when it was presented after extensively trained CSs. Perhaps the simplest version of this view is that, in consistency with models such as the Rescorla-Wagner (1972) model, after the briefly trained CS the US is processed more extensively because it is less expected. That more extensive processing of the US may include activation of sensory-perceptual processes that could mediate learned taste aversions, processing that might be absent when a well-predicted food US is delivered after an extensively trained CS.

From this perspective, the critical determinant of mediated learning potential is not the amount of training per se but, rather, the greater processing of surprising USs that is guaranteed by small amounts of training. Thus, if the surprise value of food presentations could be maintained despite extensive training of a CS, that CS might be able to mediate aversion learning. At first glance, it would appear that the partial reinforcement of a CS (as in Experiment 3) should maintain processing of the US on reinforced trials over a larger number of US presentations, and hence should enhance mediated learning. We found no such evidence. However, it is notable that the PRF and CRF schedules supported similar, high levels of CRs after extensive training in Experiment 3, which suggests that the expected value of the US did not differ much between those two schedules. Thus, on reinforced trials the US would have been similarly processed in both conditions. Note too that the high level of US expectancy in the PRF condition would tend to maximize the surprise effect of the omission of the US on nonreinforced trials in the PRF condition, increasing the chance of enhancement of processing of the CS on those trials, as was intended in Experiment 3 and as was observed by Pearce and his colleagues (Collins \& Pearce, 1985; Collins et al., 1983).
Perhaps extensive training with a lean schedule of partial reinforcement, or indeed any manipulation designed to keep the associative strength of a CS low, might permit the maintenance of mediated learning ability over extensive training of a CS. Likewise, this ability might be restored to an extensively trained CS if the US were made surprising again. For example, an extensively trained CS might be reinforced while accompanied by a separately trained conditioned inhibitor, or after an equally extensive period of extinction. On the other hand, if sensory aspects of CS-food learning are retained in extinction (see, e.g., Rescorla, 1996a), the CS might not reacquire this ability despite the overall surprise value of the reinstated reinforcer, because the sensory aspects of that reinforcer would remain expected.

Regardless of the mechanisms through which it occurs, the change in the ability of associatively activated event representations to participate in the new learning observed here over the course of training is consistent with the claims of many other researchers who have suggested that the contents of learning change over the course of training. Common to most of these views is the notion that learned performance is more flexible and goal oriented in early stages but becomes increasingly automatic and less governed by its consequences with more extensive training (see, e.g., Allport, 1937; Kimble \& Perlmuter, 1970; Tolman, 1948). For example, Adams and Dickinson (1981) suggested that extensive training may be accompanied by a shift from behavioral control by stimulus-reinforcer or response-reinforcer associations to control by stimulus-response associations. This view is echoed in several recent investigations of the neurobiological bases of learning. For example, the results of a series of investigations by Packard and his colleagues (e.g., Packard \& McGaugh, 1996; Poldrack \& Packard, 2003) indicate that the behavior of rats in plus mazes is controlled primarily by hippocampus-dependent learning of place-food relations early in training, but it becomes more controlled by caudate-dependent stimulus-response learning as training progresses. Similarly, Bussey et al. (1996) attributed initial stimulus-reinforcer learning in a conditional discrimination task to anterior cingulate systems, and later stimulus-response learning to posterior cingulate systems.

Implicit in many of these views is the idea that CSs lose access to stimulus aspects of a memorial representation of the reinforcer as training progresses. Within the context of the present results, early in training the CS activates a representation of food that not only mediates the performance of food-related CRs but also can substitute for food itself in the establishment of a learned aversion; later in training, the CS no longer activates such a representation. However, in both Experiment 3 and Holland (1998, Experiment 3), despite the inability of extensively trained CSs to produce mediated aversion learning, those CSs clearly maintained their associative access to a representation of food, because their food-cup-approach CRs were still sensitive to reinforcer devaluation. Thus, the no- 
tion that the CSs activate a unitary "food representation" seems untenable. Instead, it seems reasonable to suggest that reinforcer devaluation and representation-mediated learning effects involve different aspects of associatively activated event representations, and that associative access to those various representational features changes over the course of training.

Holland $(1990,1998)$ proposed that representationmediated learning and representation-mediated performance of CRs (as illustrated in reinforcer devaluation effects) reflect the associative activation of perceptual and motivational processing systems, respectively. In an extreme version of the former case, Holland (1990) suggested that mediated food aversion learning might occur because the CS activates perceptual processing normally activated by the food itself, as if, for example, the tone induced tasting of the food reinforcer. Holland (1990) presented initial behavioral evidence to support that claim, recording oral/facial taste reactivity measures (Berridge, 2001; Grill \& Norgren, 1978). In one experiment (Holland, 1990, Experiment 1A), thirsty rats first received pairings of two auditory CSs with the delivery of two differently flavored sucrose solutions to a liquid cup in a standard experimental chamber. An aversion to one of those solutions was then formed by pairing it with $\mathrm{LiCl}$ injection in the absence of the CSs in the rats' home cages. Finally, the rats were allowed to drink plain water in the liquid cups, and each of the auditory CSs was presented. The rats displayed more aversive oral/facial responses characterized as "disgust" or "disliking" responses when the auditory CS whose flavor associate had been devalued was presented than in the presence of the other CS or no $\mathrm{CS}$, and more appetitive or "liking" responses when the auditory CS whose flavor associate had not been devalued was presented. Because these responses are often claimed to reflect reactivity to taste properties of stimuli and are not necessarily correlated with the amount of consumption (Berridge, 2001), we suggested that their emergence to plain water in the presence of the two auditory CSs reflected associatively activated "tasting."

A second experiment (Holland, 1990, Experiment 3) provided more compelling evidence of this associative control of perceptual taste processing by auditory CSs. Water-deprived rats first received pairings of tone and noise CSs with sucrose or saline solutions. Next, the rats were given taste aversion training in their home cages through a negative-pattern discrimination procedure. In this procedure, each flavor alone was paired with toxin, but a sucrose + saline compound was nonreinforced. Finally, the rats were allowed to drink plain water in the experimental chambers. As with the CS whose flavor associate had been devalued in the previous experiment, when either CS was presented alone the rats displayed aversive responses, as would be appropriate to the individual sucrose and saline solutions. However, when the noise and tone were presented simultaneously for the first time, the rats displayed predominantly appetitive responses, as would be appropriate to the taste of the sucrose + saline compound. It is difficult to interpret this pattern of results without recourse to some control of taste processing by the auditory CSs alone.

In a more recent experiment (Kerfoot, Lee, Agarwal, \& Holland, 2004, 2005) similar to that of Holland (1990, Experiment 1A), we provided some evidence for this notion from neural systems. In a single training session, food-deprived rats first received a tone CS and the delivery of a sucrose solution through an intraoral cannula. For some of these rats, the tone and sucrose were paired, and for others those events were explicitly unpaired. Then, half of the rats in each of the groups were made ill immediately after intraoral infusions of sucrose alone, and the rest were made ill 6-8 h later. Finally, in a subsequent session, the tone was presented with intraoral infusion of plain water in all of the rats. The rats that had previously received tone-sucrose and then sucrose-illness pairings displayed aversive responses, whereas the rats that had received tone-sucrose pairings but unpaired sucrose and illness displayed appetitive responses. Rats that had not received tone-sucrose pairings displayed neither appetitive nor aversive responses in the test. The perceptual origin of these responses was suggested by the results of an analysis of patterns of brain activation. Immediately after the test sessions, the rats were sacrificed and their brains assayed for expression of FOS protein as an indicant of neural activity during the tone--water test. More FOS was expressed in gustatory sensory regions in the rats that had previously received tone-sucrose pairings, regardless of devaluation training. By contrast, more FOS was observed in brain regions associated with appetitive and aversive taste reactivity (anterior and posterior accumbens shell, respectively; Reynolds \& Berridge, 2002) in the tonesucrose nondevalued rats and the tone-sucrose devalued rats, respectively. It would be worthwhile to determine whether these devaluation-sensitive taste reactivity responses to the tones would also be observed only after minimal training, such as that given in these studies, and not after more extensive tone-sucrose training. Finally, it is notable that analogous findings have been reported in humans; for example, in an fMRI study, McIntosh, Cabeza, and Lobaugh (1998) reported activation of primary visual cortex by auditory cues that were consistently paired with visual stimuli. Interestingly, these authors did not report that the participants actually experienced the predicted but absent auditory cues, as was found in some earlier studies (Howells, 1944; Leuba, 1940). Thus, the extreme stance (following, e.g., Konorski's [1967, p. 174] discussion of CS-elicited "hallucinations" of USs) that the rats in our studies actually "tasted" the absent flavors is unnecessary as long as the CSs controlled neural processing that permitted association of associatively activated sensory information with illness.

By contrast, as evidenced in devaluation procedures, representation-mediated performance of food cup entry responses, which persists over extensive training, may reflect a CS's continued access to more downstream aspects of US processing systems. Because such devalua- 
tion effects are CS and US specific (see, e.g., Colwill \& Motzkin, 1994; Holland, 1990, 1998), these systems must include the association of sensory properties with systems that attach motivational significance to events (see, e.g., Balleine \& Dickinson, 2000; Blundell et al., 2001), but they may not provoke sensory processing sufficient to mediate learning about those absent events. For example, in a recent model of amygdalocortical interactions in outcome expectancy learning based in part on the results of electrophysiological recordings of neuronal activity and devaluation experiments (Holland \& Gallagher, 2004), neurons in the basolateral amygdala that encode specific outcome expectancies are needed to "train" similar coding in neurons in the orbitofrontal cortex. These cortical neurons may later guide performance independently of the amygdala. In such models, there is potential for the reencoding of sensory information in systems that mediate performance but that may not support the kinds of mediated learning discussed in this article.

\section{REFERENCES}

AdAms, C. D. (1982). Variations in the sensitivity of instrumental responding to reinforcer devaluation. Quarterly Journal of Experimental Psychology, 34B, 77-98.

Adams, C. D., \& Dickinson, A. (1981). Actions and habits: Variations in associative representations during instrumental learning. In N. E. Spear \& R. R. Miller (Eds.), Information processing in animals: Memory mechanisms (pp. 143-165). Hillsdale, NJ: Erlbaum.

Allport, G. W. (1937). Personality: A psychological interpretation. New York: Holt.

Balleine, B. W., \& Dickinson, A. (1998). Goal-directed instrumental action: Contingency and incentive learning and their cortical substrates. Neuropharmacology, 37, 407-419.

Balleine, B. W., \& Dickinson, A. (2000). The effect of lesions of the insular cortex on instrumental conditioning: Evidence for a role in incentive memory. Journal of Neuroscience, 20, 8954-8964.

BerRIDGE, K. C. (2001). Reward learning: Reinforcement, incentives, and expectations. In D. Medin (Ed.), The psychology of learning and motivation (Vol. 40, pp. 223-278). San Diego: Academic Press.

Blundell, P., Hall, G., \& Killcross, S. (2001). Lesions of the basolateral amygdala disrupt selective aspects of reinforcer representation in rats. Journal of Neuroscience, 21, 9018-9026.

Bussey, T. J., Muir, J. L., Everitt, B. J., \& Robbins, T. W. (1996). Dissociable effects of anterior and posterior cingulate cortex lesions on the acquisition of a conditional visual discrimination: Facilitation of early learning vs. impairment of late learning. Behavioural Brain Research, 82, 45-56.

Collins, L., \& Pearce, J. M. (1985). Predictive accuracy and the effects of partial reinforcement on serial autoshaping. Journal of Experimental Psychology: Animal Behavior Processes, 11, 548-564.

Collins, L., Young, D. B., Davies, K., \& Pearce, J. M. (1983). The influence of partial reinforcement on serial autoshaping with pigeons. Quarterly Journal of Experimental Psychology, 35B, 275-290.

Colwill, R. M., \& Motzkin, D. K. (1994). Encoding of the unconditioned stimulus in Pavlovian conditioning. Animal Learning \& Behavior, 22, 384-394.

Dickinson, A., \& Mackintosh, N. J. (1979). Reinforcer specificity in the enhancement of conditioning by posttrial surprise. Journal of Experimental Psychology: Animal Behavior Processes, 5, 162-177.

Dwyer, D. M. (2003). Learning about cues in their absence: Evidence from flavour preferences and aversions. Quarterly Journal of Experimental Psychology, 56B, 56-67.

Grill, H. J., \& Norgren, R. (1978). The taste reactivity test. I. Mimetic responses to gustatory stimuli in neurologically normal rats. Brain Research, 143, 263-279.
Hall, G. (1996). Learning about associatively activated stimulus representations: Implications for acquired equivalence and perceptual learning. Animal Learning \& Behavior, 24, 233-255.

Hall, G., \& Pearce, J. M. (1979). Latent inhibition of a CS during CS-US pairings. Journal of Experimental Psychology: Animal Behavior Processes, 5, 31-42.

Holland, P. C. (1981). Acquisition of representation mediated conditioned food aversions. Learning \& Motivation, 12, 1-18.

Holland, P. C. (1983). Representation mediated overshadowing and potentiation of conditioned aversions. Journal of Experimental Psychology: Animal Behavior Processes, 9, 1-13.

Holland, P. C. (1990). Event representation in Pavlovian conditioning: Image and action. Cognition, 37, 105-131.

Holland, P. C. (1998). Amount of training affects associativelyactivated event representation. Neuropharmacology, 37, 461-469.

Holland, P. C., Bashaw, M., \& QuinN, J. (2002). Amount of training and stimulus salience affects associability changes in serial conditioning. Behavioural Processes, 59, 169-183.

Holland, P. C., \& Forbes, D. T. (1982a). Control of conditional discrimination performance by CS-evoked event representations. Animal Learning \& Behavior, 10, 249-256.

Holland, P. C., \& Forbes, D. T. (1982b). Representation mediated extinction of flavor aversions. Learning \& Motivation, 13, 454-471.

Holland, P. C., \& Gallagher, M. (2004). Amygdala-frontal interactions and reward expectancy. Current Opinion in Neurobiology, 14, 148-155.

Holland, P. C., \& Rescorla, R. A. (1975). The effect of two ways of devaluing the unconditioned stimulus after first- and second-order appetitive conditioning. Journal of Experimental Psychology: Animal Behavior Processes, 1, 355-363.

Holland, P. C., \& Straub, J. J. (1979). Differential effects of two ways of devaluing the unconditioned stimulus after Pavlovian appetitive conditioning. Journal of Experimental Psychology: Animal Behavior Processes, 5, 65-78.

Howells, T. H. (1944). The experimental development of color-tone synesthesia. Journal of Experimental Psychology, 34, 87-103.

Kaye, H., \& Pearce, J. M. (1984). The strength of the orienting response during Pavlovian conditioning. Journal of Experimental Psychology: Animal Behavior Processes, 10, 90-109.

Kerfoot, E. C., Lee, H. J., Agarwal, I., \& Holland, P. C. (2004). Conditioned auditory stimuli control appetitive and aversive tastereactivity responses in a devaluation task (Program No. 206.10) [Online]. 2004 Abstract Viewer/Itinerary Planner. Washington, DC: Society for Neuroscience.

Kerfoot, E. C., Lee, H. J., Agarwal, I., \& Holland, P. C. (2005). Control of neural FOS and taste-reactivity responses by auditory stimuli in a devaluation task. Manuscript submitted for publication.

Kimble, G. A., \& Perlmuter, L. C. (1970). The problem of volition. Psychological Review, 77, 361-384.

KonORSKI, J. (1967). Integrative activity of the brain. Chicago: University of Chicago Press.

LEUBA, C. (1940). Images as conditioned sensations. Journal of Experimental Psychology, 26, 345-351.

McIntosh, A. R., Cabeza, R. E., \& Lobaugh, N. J. (1998). Analysis of neural interactions explains the activation of occipital cortex by an auditory stimulus. Journal of Neurophysiology, 80, 2790-2796.

PACKARD, M. G., \& McGaugh, J. L. (1996). Inactivation of hippocampus or caudate nucleus with lidocaine differentially affects expression of place and response learning. Neurobiology of Learning \& Memory, 65, 65-72.

Pearce, J. M., \& Collins, L. (1987). An evaluation of the associative strength of a partially reinforced serial CS. Quarterly Journal of Experimental Psychology, 39B, 273-293.

Pearce, J. M., \& Hall, G. (1980). A model for Pavlovian learning: Variations in the effectiveness of conditioned but not of unconditioned stimuli. Psychological Review, 106, 532-552.

Pearce, J. M., Kaye, H., \& Collins, L. (1985). A comparison of the effects of partial reinforcement schedules using a within-subject serial autoshaping procedure. Quarterly Journal of Experimental Psychology, 37B, 379-396. 
Pearce, J. M., Redhead, E. S., \& Aydin, A. (1997). Partial reinforcement in appetitive Pavlovian conditioning with rats. Quarterly Journal of Experimental Psychology, 50B, 273-294.

Poldrack, R. A., \& PACKARD, M. G. (2003). Competition among multiple memory systems: Converging evidence from animal and human brain studies. Neuropsychologia, 41, 245-251.

RESCORLA, R. A. (1992). Response-outcome versus outcome-response associations in instrumental learning. Animal Learning \& Behavior, 20, 223-232.

Rescorla, R. A. (1996a). Preservation of Pavlovian associations through extinction. Quarterly Journal of Experimental Psychology, 49B, 245-258.

RESCORLA, R. A. (1996b). Response-outcome associations remain functional through interference treatments. Animal Learning \& Behavior, 24, 450-458.

RESCORLA, R. A. (1999). Within-subject partial reinforcement extinction effect in autoshaping. Quarterly Journal of Experimental Psychology, 52B, 75-87.

Rescorla, R. A., \& Wagner, A. R. (1972). A theory of Pavlovian conditioning: Variations in the effectiveness of reinforcement and nonreinforcement. In A. H. Black \& W. F. Prokasy (Eds.), Classical conditioning II (pp. 64-99). New York: Appleton-Century-Crofts.

Reynolds, S. M., \& Berridge, K. C. (2002). Positive and negative motivation in nucleus accumbens shell: Bivalent rostrocaudal gradients for GABA-elicited eating, taste "liking"/“disliking" reactions, place preference/avoidance, and fear. Journal of Neuroscience, 22, 73087320

Tolman, E. C. (1948). Cognitive maps in rats and men. Psychological Review, 55, 189-208.

Trapold, M. A., \& Overmier, J. B. (1972). The second learning process in instrumental training. In A. H. Black \& W. F. Prokasy (Eds.), Classical conditioning II (pp. 427-452). New York: Appleton-CenturyCrofts.

(Manuscript received November 4, 2004; revision accepted for publication February 22, 2005.) 